\title{
Radium and Radio-Activity-II
}

\author{
A Consideration of Some of the Physical and Chemical Aspects \\ By Charles H. Viol \\ [Continded from Scientific American Supplement, No. 2256, Page 195, March 29, 1919]
}

THE work of a great many experimenters has shown that these active deposits are complex, consisting of a mixture of various successive radio-active products The various products in an active deposit exhibit char acteristic chemical and physical properties and, by taking advantage of these, separations of the various forms of radio-active matter in the deposit may be effected. These products are always present in unweigbably small quantities (due to their short periods) and so these separations must always leave the radioactive material mixed with certain quantities of ordinary inactive matter necessary in order that there nary inactive matter necessary in order that there
may be something tangible with which to work. The may be something tangible with which to work. The active deposit of thorium, collected on a platinum plate plate to a white heat. In the radium series the first product from the emanation, called radium $\mathbf{A}$, has a period of three minutes. This radium $A$ in disintegrat Ing forms what is called radium $B$, a product that has a period of 26.8 minutes. Radium $B$ gives rise to beta rays and the product radium C. Radium C, decaying with a period of 19.5 minutes, produces alpha, beta and gamma rays and radium $D$. Radium $D$ has a longe period, 16.5 years, so that while the active deposit on substance which has been exposed to radium emanation at first decays rapidly-due to the short periods of radium $\mathrm{A}, \mathrm{B}$, and $\mathrm{C}$-there is left a slight residual actlvity, due to the slow decaying $D$, and the subsequent products radium $\mathrm{E}$ and radium $\mathrm{F}$. The period of radium $E$ is five days, and that of radium $F$ is 13 days. Radium $F$ gives off alpha rays, and we now know that the product which Mme. Curie first isolate with the bismuth from pitchblende residues, and called polonium, is nothing other than radium $F$, that accumulates in the pitchblende.

The radio-elements now known number about thirty, and may be grouped into three families-the uranium radium group, the actinium group and the thorium group Since the half-decay period of radium is about 1700 years, it is evident that in about 20,000 years prac tically all the radium now existing will have transmuted, and, in order to account for the presence of radium today, a longer-lived product must be sought. This parent is the element uranium. A number of researches, "beginning with the work of Boltwood, McCoy, and of Strutt, have shown that there is a relaCoy, and of Strutt, have shown that there is a relation between the amount of radium and of uranium in
most uranium minerals. Experiments were carried out to test whether the newly-formed radium could be detected in uranium initially freed from radium. The experiments gave negative results, and Boltwood found that there is a long-lived product, ionium, in the series between uranium $\mathrm{X}$ and radium.

Actinium is always found in the uranium minerals, and its origin is obscure, though it seems probable that it results as a branch product in the uranium series. That is, one of the products in the uranium series is thought to disintegrate in two fashions, one of the products being actinium, the product of the other method of changing being a substance which finally leads to radium The accompanying diagrammatic representation of these series of radio-active elements shows the order in which the products occur, their half-decay periods, the rays which they give off in the process of their transmutation, and their maximum valence (Roman numeral).

All of the decay products of the uranium-radium series are found in any geologically old ore containing uranium, the proportionate amount of each product deponding upon the rate at which the radio element is undergoing the process of transmutation or decay. For each three million parts of uranium in an old unaltere ore, there is one part of radium. Consequently for each per cent. of uranium oxide $\left(\mathrm{U}_{3} \mathrm{O}_{8}\right)$ in a ton of uranium ore there are 25 milligrams of radium element. Radium of the series has come to be of mortance than the other members of the series, owing to the fact that it is undergoing the transmutation process rather slowly, requiring 1700 years for the decay of half of any quantity, while the subsequent products, Radium Fmanation, $\mathrm{Ra} \mathrm{A}, \mathrm{Ra} \mathrm{B}$ and the complex $\mathrm{Ra} \mathrm{C}$, are all undergoing a very rapid process of transmutation, resulting in the rapid emission of a succession of rays from from each of these products for each atom of radium that tranforms. Thus, a radium prepation has the long life of radium, and the high degree of radio-ac tivity contributed by the rapid transmutation of the successive decay products of radium. No other radioelement combines these two advantages in the same degree, the nearest competitor, mesothorium, having a far shorter life than radium, the time required for the transmutation of half of any amount of this element being only 5.5 years.

Pitchblende, the ore from which radium was first produced, occurs in small quantities in several locali-
ties in the United States, notably in Gilpin County,
Coloraclo, but so far this ore has not been the source of any radium produced in this country. Carnotite, a potassium uranyl vanadate, is the ore which occurs most extensively in the United States, the most notable deposits being those of southwestern Colorado and southeastern Utah. High-grade carnotite, which is comparatively rare, is a soft, talc-like sulphur-yellow mineral, corresponding essentially to the composition of a potassium uranyl vanadate. The commercial carnotite ore is a low-grade mineral usually found in sandstone formations where the carnotite material acts as a cement for the grains of sand. This low-grade ore contains from traces up to several per cent. of uranium oxide, the workable ore containing from about 0.5 per oxide, the workable ore containing from about 0.5 per
cent. upward. Owing to the greater amounts of lowergrade ore, the average working ore contains about 1 per cent. of uranium oxide, and consequently each ton of this ore contains 2.5 milligrams of radium element. The great problem of radium production is the economical treatment of these lower-grade ores, which must be conserved because of their more extensive occurrence. The region in which carnotite is mined is remote from railroads, and so the ore must be hauled long distances by burros and wagons. Burros bring the ore from the hills to the wagon roads and the average wagon haul is about forty miles. The ore as found usually consists of a thin layer of sandstone which crops out on the side of a canyon wall and is recognized by the characteristic sulphur-yellow color. The narrowness of the seams makes it necessary to take out a large amount of worthless stone to secure the carnotite ore. The seams are usually in the form of pockets, so that the value of a claim is dubious until it has been thoroughly explored and worked.

The physical nature of low-grade carnotite ore is such as to make possible a mechanical concentration of the values. This is carried out on ore containing 2 per cent. or less of uranium oxide. The methods in use are both wet and dry, and consist essentially in a process of grinding to loosen the carnotite material from the sand grains and then separating the values from the tails by a process of dry or wet elutriation.

The fine powdered concentrates are next subjected to chemical treatment to remove the uranium and vanadium, and then the residue, which still contains the radium, is subjected to further chemical treatment to remove this constituent together with the barium salts present in the ore.

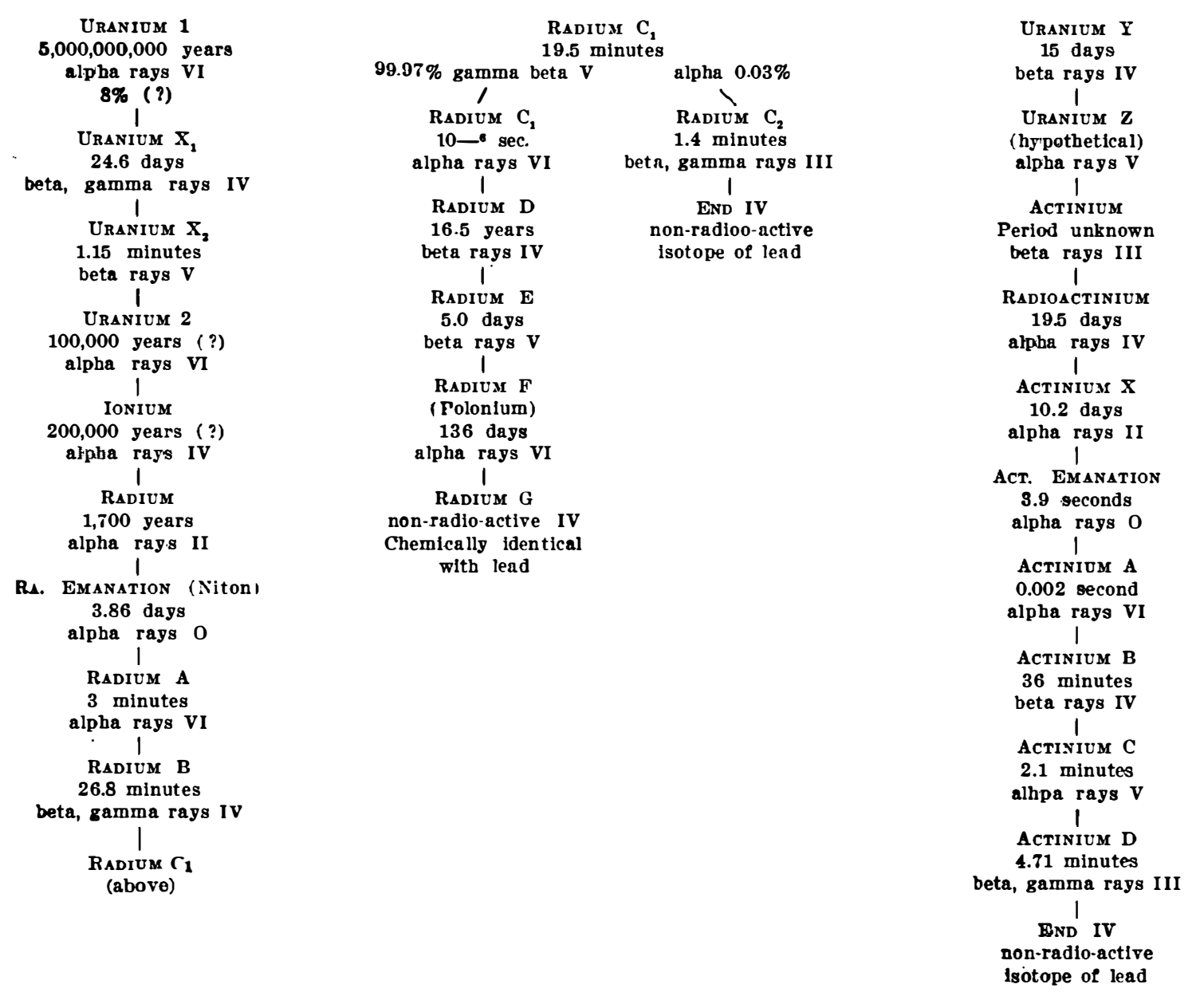

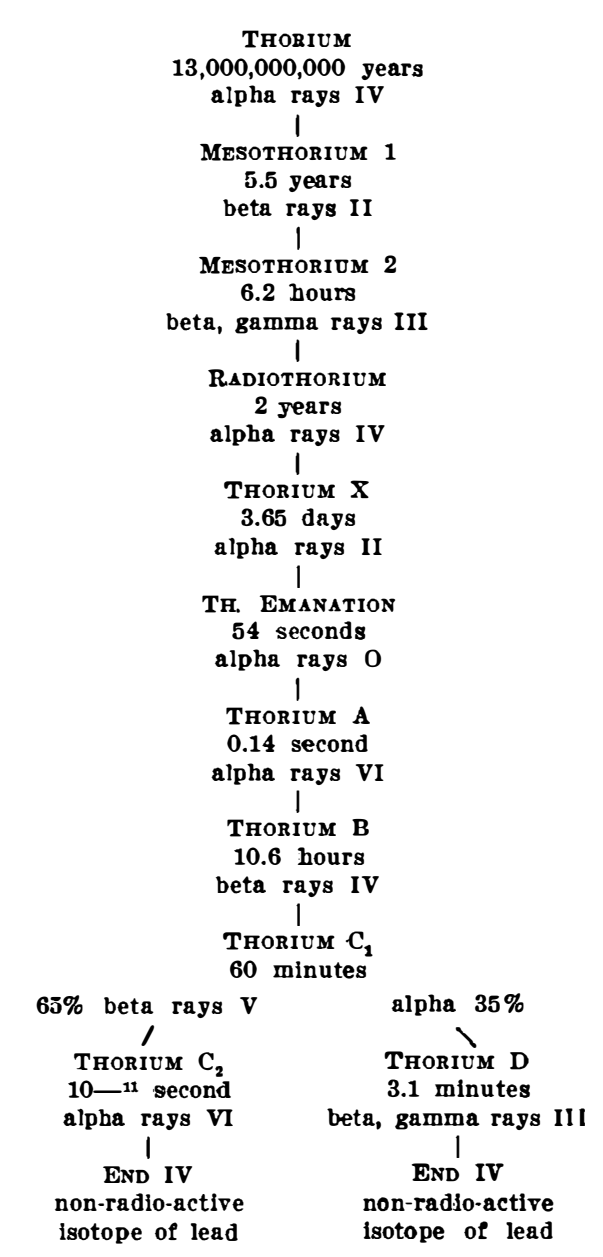

TABLE OF THE RADIO-ELEMENTS 
Chemically, radium is so nearly like barium as to make it impossible to separate the radium from the barium present in the ore by a single operation. Since a ton of average ore contains only a few milligrams of radium, while the ore usually contains in the order of a half per cent. of barium sulphate or other barium salts, it is obviously simpler to regard the chemical treatment of the ore as a process of removing all the barium from the ore. This is carried out in a number of dif ferent ways; some wet, as in the Bureau of Mine nitric acid treatment, which is only applicable to coarsely-ground, high-grade ore, or boiling the concen-
trates with soda, with subsequent acid leaching; or dry methods, such as fusion with alkali or soda, with subsequent solution of the insoluble radium-bariumcalcium residue in acid.

The next step in the preparation of radium salt consists in subjecting the radium-barium salt to a process which will separate the radium from the barium. No strictly chemical process for effecting thi separation is suitable, and practically a process of fractional crystallization of the chloride, bromide or hydroxide is used. It was found by Mme. Curie, for example, that radium-barium chloride, when allowed to crystallize from an aqueous solution, gives crystals containing a greater proportion of radium to barium than was contained in the original salt, or the salt left In solution in the mother liquor. Application of this fact in a process of fractional crystallization makes 1 possible to concentrate the radium in the "heads" and the barlum in the "tails," resulting finally after hun dreds of operations in the production of a few hundred milligrams of comparatively pure radium salt from the treatment of hundreds of kilos of the crude radium barium salt as obtained from the ore

There is probably a total of not more than a hun dred and twenty grams of radium element in all the high-grade radium preparations in the whole world. Of this amount thirty grams have been prepared in the Radium Research Laboratory of the Standard Chemlcal Company during the five years that production has been under way. Other production in the United States, including the radium produced by the United States Bureau of Mines for the National Radium Institute, probably totals about fifteen grams, making the United States the producer, to date, of over a third of the word's radium supply. The present producing capacity of the Standard Chemica

to the present has been in the treatment of various growths, malignan and benign. Certain types of cancer respond readily to the action of the beta and gamma rays of radium (the more penetrating rays) and this same action also enables the medical man to give great relief in certai types of hopeless cancer, where medical science has heretofore been helpless. For these medical purposes the radium salt is used, not as a medicine but rather as a physical agent for the production of its characteristic rays. The radium, usually in the form of radium barium sulphate, containing from 60 per cent. to 90 per cent. of pure radium sulphate, is either sealed hermetically in tiny thin-walled glass tubes or is sprea in an enamel or varnish over the surface of a metallic plaque. In this way the radium salt is comparatively practically no depreciation, since the activity falls by only one per cent. in about twenty-five years.

A use of radium which has a considerable application, and which now is becoming of almost as great importance as the medical use, is in the production of the so-called permanently luminous compounds. Thi luminous material finds a wide range of use in all darkness or seml-darkness.

Phosphorescent compounds are those which when exposed to suitable light are capable of storing up some of the energy, which is subsequently emitted in the form of light. A number of phosphorescent substance are known in which the luminescence persists for som time. Of these, the best known was the specially pre-
pared calcium sulphide, which formed the base of Balmain's patented lumine, which formed the base of ure to light is absolutely essential to the production of any degree of luminescence, and after such exposure this material emits a bluish light for only several hours. It was first used about 1877 on watch and clock dials, It was first used about 1877 on watch and clock dials,
etc., but was practically discarded about twenty years ago, largely because of the necessity of first exposing the articles to light to render the luminous paint effective.

Early in this country, after the discovery of radium and other strongly radio-active substances, such as actinlum, it was found that the rays of the radio-active substances were capable of exciting certain materials to the emission of visible light, even when not previ- ously exposed to light-the necessary energy being supplied from the radio-active material. Of all known materials, prosphorescent zinc sulphide, so-called "Sict's hexagonal blende," gives the greatest luminthe light from the zinc sulphide being due to the numerous scintillations produced by the bombarding the zinc sulphate crystals by the alpha rays.

Phosphorescent zinc sulphide consists of a specially prepared crystalline form of zinc sulphide which, when mixed with an amount of radium, continues to emit a greenish-yellow light, the intensity of the luminesence being dependent on the quality of the zinc sulphide and the proportion of radium used.

It has been known that many phosphorescent subtances respond strongly to the action of ultra-violet rays and X-rays; however, the substances which are very luminescent under the action of these rays (such for example, as Balmain's calcium sulphide, and native willemite) do not necessarily produce the brightest luminous mixtures when radium is added. To test the suitability of any given material, it is either necessary to add radium directly to the substance and compare the resulting luminosity with that of a good grade of zinc sulphide containing an equivalent amount of radium, or otherwise to bring alpha rays to play on the materials. We have found a polonium plate about twenty millimetres in diameter most useful, since this preparation emits an intense alpha radiation. With a suitable polonium plate a superficial luminescence can be obtained in zinc sulphide equal to that obtained by the addition of several hundred micrograms of radium per gram of zinc sulphide. By holding this plate over two adjacent specimens of zinc sulphide or other material, it is very easy to determine the relative values of their alpha-ray luminescence.

The luminescence of the zinc sulphide is roughly proportional to the radium content, falling off someadded. Commercially luminous compound is in use containing from 25 to 300 micrograms (millionths of a gram) of radium element per gram of the mixture. Careful photometric tests have demonstrated that the rate of decay of the luminescence is proportional to the amount of radium, so that compounds which initially are brightest show the most rapid falling off in luminescence, while compounds containing proportionally less radium are not initially so brilliant, but have a longer effective life.

The decay in the luminescence of compounds in which radium is the exciting agent is not due to change in the radio-active substances, since it is well known that radium changes so slowy as to require 1,700 years for its half-decay-approximately 1/25th of one per cent. change taking pace in one year. The change in the zinc suphide, however, is not slow, which acpound with time. There are many points in connection with light emission from zinc sulphide that still remain to be explained. However, it may be said that the behavior of the substance indicates a capacity for sending out only a certain total amount of light, so that where a greater luminosity is excited by the use of a larger proportion of radium, the life of the zinc sulphide is proportionately shortened.

In the application of the luminous compound for its various uses it is mixed with a suitable vehicle, usually some form of clear. transparent varnish, the resulting thick paint being applied by means of a fine stiff-haired brush or a fine stylus. The difficulties to be overcome in this connection are dependent on the nature of the material and surface to be painted, as well as the nature of the surroundings. Paper, porcelain, mica, and metal dials of all sorts bearing figures and lines are now used. In the liquid-damped compasses, such as the aero-compasses, the card floats in alcohol, thus introducing a further requirement that the luminous paint resist the action of this solvent. The varnish most suitable for this purpose is yet to be found. For some instruments, such as aeroplane instruments, the requirements of luminosity are of most Importance and outwelgh the other considerations of cost and length of effective life. For such purposes the grades of luminous compound are prepared which give the highest initial luminosity, due to a larger proportion of radium, and having, consequentlly, a shorter effectlve life. For many other purposes, such as the use on marching compasses, wrist watches, etc., luminous compound of lower initial luminosity is recommended, and this material has the advantage of lower cost and longer life

The chemistry of the radio-elements and the work most important an of the atom (a large number of the in the years. The article by Rutherford on "The Structure of the Atom" in vol. 27, p. 488, March, 1914, is very interesting for the general reader) which has grown out of the study of the radio-elements and their rays presents a far more interesting field than the technology of radium, particularly since the various phases of the treatment of radium ore, etc., cannot be detailed minutely. The atom, according to J. J. Thompson, can be considered as a series of concentric rings of electrons in rapid orbital motion about or within a spher of positive electrification. Such an atom is capable of accounting for many of the properties of matter, both material and electrical. Rutherford more recently has modified this view of the atom and considers the sphere of positive electrification or nucleus of the atom as exceedingly minute in comparison with the dimensions of the atom. This view is the result of careful observations on the scattering or deviation of alpha rays from their paths in passing through matter, the assump tion being that the very sharp bends in the path of the alpha ray indicate an approach of the alpha ray to the nucleus, causing the deviation. Mathematical calculations by Darwin for alpha rays in hydrogen demonstrate that the centres of the nuclei (the alpha ray is the nucleus of the helium atom) must approach to within $1.7 \times 10^{-1 s}$ centimetres of each other. This view is confirmed also by experiments made by Marsden on the repulsion of the hydrogen nuclei by alph rays. According to Rutherford, the hydrogen atom consists of one negative electron moving orbitally about the nucleus, which consists of a positive electron having a much smaller diameter than the negative electron is about $1 / 1700$ the mass of the hydrogen atom, so that the bulk of the mass of the hydrogen atom must reside in the nucleus, and this is accounted for on the basis of electro-magnetic mass by the fact that the dlameter of the hydrogen nucleus is so muc smaller than that of the negative electron. Alpha rays penetrating into a gas, such as hydrogen, move through a definite distance before they lose their kinetic energy and become ordinary helium atoms. This distance is called their range, and within its range the alpha ray is capable of producing such phenomena as onization of gases, scintillation in zinc sulphide, etc whereas at a distance greater than the range, thes phenomena are not observed. Marsden has found that when the alpha rays from radium impinge Into hydrogen gas there are the usual scintillations observed on a zinc sulphide screen due to the alpha rays; however, beyond the range of the alpha rays, some three or four times the distance to which the alpha rays could penetrate, there are still scintillations and these are attributed to the hydrogen nuclei which are set in rapid motion by collision with the alpha rays, the energy of the alpha ray being transferred to the hydrogen nucleus, and this having a smaller mass moves through the longer range.

$$
\text { [TO BE CONTINUED] }
$$

"Protactinium," a New Radio-Active Element Acconding to a note in the Chemical Trade Journal for June 29th, a new radio-active element of considerable emissive power has been detected in the residue from pitchblende, which forms the raw material employed as a source of radium. This residue was subjected to treatment which finally left undissolved only the members of the tantalum group; and this insolubie remainder showed a radiation, at first slight, but gradually increasing largely, which proceeded mainly from the evolution of actinium, and indicated the presence of the new element "protactinium." Experiments for the separation of the elements are to be undertaken. The period of semi-disintegration probably fluctuates between statements published in the Munchner Neueste Nachrichten.-Nature.

\section{Lateral Deviation of Projectiles}

K. H. GÜLDNER, in the Zeitschrift des Vereines deutocher Ingenieure for August 11th and 18th, 1917, describe some investigations which he carried out to determine the lateral deviation of projectiles caused by the spin mparted to them by the rifling of a trench-mortar. Th trench-mortar provides a suitable means of carrying out such tests as the motion of the projectile may be followed by the eye. Rifling with a right-handed twist may cause constant lateral deviation both to the right and left. Right or left deviation is the result of right left precession, and is visible to the naked eye. Left precession with rifling having a right-handed twist can occur only after the maximum height of the trajectory has been passed if the center of action of the air-resistance lies behind the center of gravity of the shell. The precession on the ascending part of the trajectory
slwsye greater than in the descending part. - Nature. 